\title{
Physicians' Role in Advancing Tobacco Control in China
}

Kylie J. LINDORFF ${ }^{1}$, David J. HILL ${ }^{2}$

${ }^{1}$ Quit Victoria and VicHealth Centre for Tobacco Control, Cancer Council Victoria, Melbourne, Australia;

${ }^{2}$ Cancer Council Victoria, Melbourne, Australia and International Union Against Cancer (UICC)

\section{医生在推动中国烟草控制中的作用}

\author{
南娟 丁燕 翻译 刘谦 校对
}

天津医科大学总医院, 天津市肺癌研究所, 天津市肺癌转移与肿瘤微环境重点实验室

【摘要】中国已经批准了烟草控制框架公约（Framework Convention on Tobacco Control, FCTC ), 并致力于执 行烟草控制方案, 以对抗烟草使用带来的大量不良健康和经济后果。医生需要在这场斗争中像其它国家, 如澳大利 亚等实施严厉烟草控制政策的国家一样，发挥主导作用。目前中国的问题包括医生自身的吸烟状况及其对患者影响 作用的低估，这意味着他们潜在的积极作用远未实现。医生在烟草控制中责无旁贷。首先应从他们自身的戒烟等行 为和做法开始, 如果医生为吸烟者, 则应戒烟, 并指导患者及患者家属不吸烟。提倡医院和医疗机构无烟, 亦为医 生的首要任务。作为人们试图改善自身健康状况和接受疾病治疗的医疗中心，允许吸烟的行为是完全不合理的。

责任亦在于医生工作的机构和代表医生的专业团体机构，应为医生提供有助于控制他们自己以及患者烟草使 用的信息、培训和支持。

【关键词】FCTC; 医生; 吸烟; 烟草; 烟草控制

【中图分类号】R734.2 DOI: 10.3779/j.issn.1009-3419.2010.06.012

前言

吸烟是全球可预防的导致死亡的最主要原 因，而且是全世界八大主要死因中六大死因的危 险因素 ${ }^{[1]}$ 。在中国, 多达100万 30 岁以下的男性死 于烟草使用 ${ }^{[2]}$ 。作为世界卫生组织 (World Health Organisation, WHO ) 烟草控制框架公约（Framework Convention on Tobacco Control, FCTC ) 的缔 约方，中国致力于执行烟草控制方案，以对抗烟 草使用带来的大量不良健康及经济后果。医生可 以，而且事实上需要，在这场斗争中发挥主导作 用。但是，目前的问题包括医生自身的吸烟状况 及其对患者影响作用的低估，这意味着他们潜在

Correspondence to: Kylie LINDORFF. Quit Victoria and VicHealth Centre for Tobacco Control, Cancer Council Victoria, Melbourne, 3053 Australia

Email: kylie.lindorff@cancervic.org.au

Thoracic Cancer, 2010, 1(1): 21-26. http://www.thoraciccancer.net
的积极作用远未实现。

中国的吸烟状况

中国的烟草消费大幅增加，从1970年低于 0.5 万亿 到1990年超过 1.5 万亿。1990年-2005年间，烟草消费进 一步增加至约 1.8 万亿 ${ }^{[1]}$ 。中国占世界人口的 $20 \%$, 但是 在全球约 6 万亿的烟草产量中, 中国消费约占 $37 \%{ }^{[3]}$ 。 1996年的一次全国调查发现，在中国63\%的男性和 $4 \%$ 的女性吸烟 ${ }^{[4]}$ 。截止至 2002 年, 这些全国数据并未明 显改善, 吸烟男性实际上升至 $66 \%$, 吸烟女性略降至 $3 \%{ }^{[5]}$ 。

这与美国、加拿大、英国和澳大利亚等西方国家的 趋势形成对比, 这些国家的烟草使用率在近 10 年显著降 低。某些飞速发展中的经济体, 如巴西, 通过强有力的 烟草控制政策和信息传播, 吸烟率有所降低 ${ }^{[6]}$ 。这些成 绩使人们坚信：鉴于政治承诺和各种资源, 中国的吸烟 率将会下降。 


\section{吸烟对健康的影响}

吸烟对健康的严重危害是冊庸置疑的。吸烟与肺癌 相关的证据出现于 20 世纪 50 年代 ${ }^{[7]}$, 而且在 40 余年前, 肺癌被断定为由吸烟引起 ${ }^{[8]}$ 。吸烟是心脏疾病、中风和 呼吸系统疾病的诱因, 而且大量的研究显示, 吸烟是越 来越多的癌症的病因 ${ }^{[9]}$ 。

烟草是肺癌的主要诱因, 全球评估显示, 烟草引起 的肺癌占全部肺癌的 $90 \%{ }^{[3]}$ 。甚至在澳大利亚等发达国 家, 肺癌患者的预后亦不良, 诊断后仅约 $11 \%$ 的男性和 $14 \%$ 的女性可存活5年 ${ }^{[9]}$ 。

烟草工业的改革包括过滤嘴的引人, 机器测定的焦 油量和毒素水平的降低，这些降低了烟草烟雾成分的暴 露, 但并未降低肺癌死亡率。这些改革可能导致吸烟者 癌症类型的改变, 与吸烟流行病早期多见的鳞癌相比, 目前诊断的癌症类型多为腺癌 ${ }^{[9]}$ 。

尽管与持续吸烟者相比, 戒烟者发展成为肺癌的 危险性降低，但戒烟者在戒除多年后，其危险性仍较从 不吸烟者高 ${ }^{[9]}$ 。吸烟导致肺癌通常需20年左右或更长时 间，因此目前肺癌的死亡率反映了 20 年-30年前公众的吸 烟率。

吸烟也是喉癌、口咽癌、食道癌、胰腺癌、胃癌、 肾癌与膀胱癌、宫颈癌和急性髓性白细胞病的诱因。亦 与肝癌和结肠 (肠) 癌明显相关。烟草烟雾中有69种已 知的致癌物质; 它们被吸人肺并通过血流运送至身体其 它部位。吸烟也影响代谢与酶的活性, 这可能影响致癌 作用 ${ }^{[9]}$ 。

\section{中国目前及预测的吸烟相关死亡状况}

1991年-2000年间，中国男性死亡的主要原因依次为 恶性肿瘤、心脏疾病、脑血管疾病、意外事故和感染性 疾病。女性死亡的主要原因依次为心脏疾病、脑血管疾 病、恶性肿瘤、肺炎和感染以及感染性疾病 ${ }^{[10]}$ 。三大主 要死因（心脏疾病、恶性肿瘤和脑血管疾病）占全部死 亡病例的 $66 \%$ 。吸烟是这三大主要死因的主要危险因素。 前五位癌症死因为恶性肺癌、肝癌、胃癌、食管癌和结 直肠癌。吸烟可引起或与前五位癌症死因均明显相关。

吸烟引起的死亡率在男性高于女性, 城市高于农 村，占总死亡率的 $7.9 \%$, 占男性死亡率的 $12.9 \%$, 占女性 死亡率的 $3.1 \%$ 。有评估认为, 如果在中国废除吸烟, 男 性总死亡率可降低 $10 \%$ ，女性总死亡率可降低 $3.5 \%{ }^{[10]}$ 。
一项全国范围内的代表性研究推算了2005年中国 吸烟导致的死亡人数 ${ }^{[11]}$ 。研究显示, 2005年, 吸烟导致 的死亡总人数为 673000 例, 其中男性538 200例, 女性 134800 例。三大主要死因为癌症（268 200例死亡）、 心血管疾病（146200例死亡）和呼吸系统疾病（66800 例死亡）。与这些死亡明显相关的特异性疾病为肺癌 （129000例死亡）、中风（20600例死亡）和慢性阻塞 性肺疾病（29200例死亡）。综合起来, 它们约占男性 吸烟导致死亡人数的 $45.1 \%$, 约占女性吸烟导致死亡人 数的 $31.8 \%$ 。这些数据并未考虑被动吸烟导致的死亡人 数。

由于中国男性吸烟率较高, 因此有人认为被动吸烟在 更广泛的公众中亦可明显引起死亡和疾病。被动吸烟可使 非吸烟成人罹患心脏疾病和肺癌, 并可促使和加剧婴儿、 儿童和成人的轻度至重度呼吸系统疾病, 增加婴儿猝死 综合症和幼童的一系列其它严重健康后果的危险性 ${ }^{[12]}$ 。 2006年，美国公共卫生署署长关于二手烟的健康后果的 报告中做出推断：没有暴露量就没有危险性 ${ }^{[13]}$ 。Gan等 进行了一项旨在评估 2002 年中国被动吸烟导致成人肺癌 的疾病负担的研究 ${ }^{[14]}$ 。他们首次估算了 2002 年主动吸烟 导致肺癌的死亡人数为 130000 例, 男性 114700 例, 女性 15300 例。随后他们估算了2002年被动吸烟导致肺癌的死 亡人数，并发现 22000 例肺癌死亡归因于被动吸烟。 $88 \%$ 的主动吸烟死亡者为男性, 与之不同, 女性在被动吸烟 中承受着巨大的压力, 占被动吸烟死亡人数的 $80 \%$ 。22 000 例死亡仅为被动吸烟导致肺癌的死亡人数。由于中国 有 4 亿被动吸烟者 ${ }^{[14]}$, 有人认为与被动吸烟相关的其它疾 病和死亡, 尤其对于女性和儿童来说, 也将增加中国吸 烟导致的重大的健康负担。

如果目前的吸烟模式持续下去, 中国的死亡人数即 可被预测, 许多研究已预测了该数字。Peto等预言, 目前 每年烟草导致的死亡约为 1 百万，2050年这个数字将会增 至约 3 百万 ${ }^{[15]}$ 。他们声明, 如果目前高的吸烟率和低的戒 烟率仍不改变, 那么在 21 世纪的前 50 年中, 中国烟草导 致死亡的人数将为 1 亿。

\section{经济影响}

在中国，吸烟的经济负担是巨大的。据评估，2000 年吸烟的经济支出为 50 亿美元 ${ }^{[16]}$ 。包括治疗吸烟相关疾 病的所有卫生保健开支在内的直接支出为 17 亿美元, 占 吸烟相关总支出的 $34 \%$, 占 2000 年中国全民保健支出的 
$3.1 \%$ 。有研究显示, 与发达国家相比, 中国处于工作年 龄的大多数人群罹患慢性疾病, 吸烟对经济的影响具有 深远意义 ${ }^{[10]}$ 。

\section{中国医生的吸烟率}

许多研究评估了中国医生的吸烟率、戒烟方式及 态度 ${ }^{[17-20]}$ 。一系列于1987年、1996年和2005年在湖北省 省会武汉实施的研究有助于我们监测不同时间段医生间 各指标的变化 ${ }^{[18,20]}$ 。男医生的吸烟率在1987年-1996年间 从 $50.9 \%$ 显著升高至 $61.3 \%, 2005$ 年略降至 $58.0 \%$ 。女医生 的数据更令人担忧。1 1987 年女医生的吸烟率仅为 $4.8 \%$, 1996年升至3倍达 $12.2 \% ， 2005$ 年再次增加超过一半达 $18.8 \%$ 。

各项研究均比较了医生的吸烟状况与其参与戒烟指 导的可能性的关系。在1987年, 医生的吸烟状况与他们 是否参与戒烟指导显著相关, 非吸烟医生明显更易于参 与对患者的指导。然而在1996年, 事实并非如此, 医生 的吸烟状况与其参与戒烟指导的可能性无关 ${ }^{[20]}$ 。在 2005 年, 非吸烟医生明显更易于参与戒烟指导, $70.5 \%$ 的非吸 烟医生经常或总是劝导其患者戒烟, 但仅 $48.6 \%$ 的吸烟医 生参与戒烟指导 ${ }^{[18]}$ 。

2004年，一项仍然在湖北省实施的研究调查了农村医 生的吸烟状况, 为城市医生与农村医生间的差异提供了一 些见解 ${ }^{[19]}$ 。研究发现, 农村男医生的吸烟率为 $31.9 \%$, 如按 性别进行分类, 农村女医生均为非吸烟者。吸烟率也随着 年龄而变化, 低于 25 岁的年轻人吸烟率最低 $(6.3 \%), 50$ 岁-54岁人群的吸烟率最高 (31.6\%)。但是, 鉴于这项研 究是在教学医院实施的, 尤其是由于年轻的医学生在医院 不允许吸烟, 因此相应的数据可能偏低 ${ }^{[18]}$ 。

吸烟状况、戒烟指导行为和态度的差异不仅存在于 农村医生和城市医生之间, 而且存在于不同城市的医生 之间。2004年一项基于6个中国城市的医生的医院调查发 现, 男性吸烟率为 $41 \%$, 女性吸烟率为 $1 \%{ }^{[17]}$ 。这些数据 明显低于湖北省武汉市医生的吸烟率。2008年一项源自 977家医院大约40 000名医生的近期研究发现, 男性吸烟 率为 $38.7 \%$, 女性吸烟率为 $1.1 \%$, 但是不同地区间吸烟率 不同 ${ }^{[21]}$ 。当对像中国这样庞大人群的国家的医生实施戒 烟支持、培训或教育时, 辨识和理解区域间的差异的意 义是成功的关键所在。

不同科室医生的吸烟率亦不同，比如外科医生、妇 科医生、儿科医生、中医医生、整形外科医生和重症监
护室医生 ${ }^{[18,19]}$ 。当执行基于医生的烟草控制计划时, 需 要考虑医生的专业方向。

\section{医生的吸烟状况对烟草控制成功的影响}

有关中国医生吸烟状况数据的持续搜集尤为重要, 因为它是象征目前烟草控制方案有效性的“晴雨表”。医 生作为健康的行为榜样, 如果持续吸烟, 就不易于说服 公众戒烟。其次, 有研究显示, 一个国家烟草流行的“成 熟度”可通过医生的吸烟率来预测, 因为他们往往比一般 人群更早戒烟 ${ }^{[22]}$ 。

澳大利亚医生吸烟率的下降为解释该现象提供了一 项实用的病例研究。在 20 世纪 60 年代, 大约 $1 / 3$ 的澳大利 亚医生吸烟, 总人口的吸烟率也较高, 超过 $1 / 3$ 的女性吸 烟, 接近 $2 / 3$ 的男性吸烟 ${ }^{[23]}$ 。在 20 世纪70年代中至晚期, 医生的吸烟率几乎减少了一半, 而且在19世纪80年代早 期, 仅约 $1 / 10$ 的医生吸烟。在 20 世纪 90 年代, 医生的吸 烟率甚至进一步降至 $1 / 20$ 。尽管在 20 世纪 90 年代, 一般 人群中男性吸烟率也有所降低, 但与医生吸烟率的下降 不一致, 一般男性吸烟率约为 $1 / 4$ 。女性吸烟率在 20 世 纪60年代至 20 世纪 80 年代起初有所升高, 但在 20 世纪 90 年代降至约 $1 / 5^{[23]}$ 。

强有力的证据显示, 医生的干预作用严重影响其 患者戒烟的可能性 ${ }^{[24]}$ 。来自澳大利亚等国家的证据也表 明, 伴随着越来越多的医生戒烟, 他们更多地参与其患 者的戒烟指导。在1964年, 不到一半的澳大利亚医生指 导其患者戒烟, 但到 1982年, 91\%的医生参与其患者戒 烟 ${ }^{[23]}$ 。因此, 当医生作为行为榜样在健康行为方面起到 更好的模范带头作用时, 他们也更易于鼓励患者不吸烟 或戒烟。

医生是影响患者的可信的和权威的资源。而且, 在 中国, 许多医生似乎并未在健康行为的榜样方面和鼓励 其患者戒烟方面发挥应有的重要作用。

据报道, 37\% 的吸烟医生在其患者面前吸烟 ${ }^{[17]}$, $51 \%$ 的吸烟医生很少或从未进行戒烟指导 ${ }^{[18]}$ 。在所有的 医生中, $21.1 \%$ 的医生对他们应该为其患者树立不吸烟的 榜样有异议, 仅 $21.4 \%$ 的医生认为医生是帮助患者成功戒 烟的最有影响力的人 ${ }^{[18]}$ 。

\section{哪些是需要做的?}

解决逐渐增多的烟草相关疾病的难题应依赖更好的

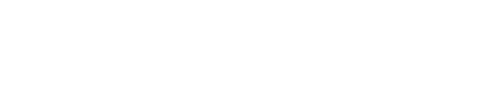


治疗还是更好的预防? 尽管将最新研究转化为更行之有 效的治疗是至关重要的, 但权威专家赞成更多地关注预 防。世界卫生组织秘书长Margaret Chan声明：治愈这一 致命疾病并非依赖药物或疫苗, 而是依赖政府和公众社 会的一致行动 ${ }^{[1]}$ 。为此, 我们还应强调: 医生需要负起 责任并发挥带头作用。

中国的研究显示, 与医生自身的吸烟状况一样, 有多 种因素与医生戒烟指导的频率显著相关。包括他们对责任 的认知、对成功的认知、对模范作用的认知以及他们是否 坚信他们在劝阻患者戒烟中具有强大的影响力 ${ }^{[18]}$ 。鼓励 和支持医生自己戒烟的教育和计划是提高他们参与患者 戒烟指导的第一步。为医生提供关于短暂干预戒烟的培 训可产生双重功效, 为鼓励医生自己戒烟提供信息和动 力, 并为其为患者解决这一问题时提供技巧和信心。

医生亦可影响吸烟行为及体制和组织层面的政策。 他们可以利用他们作为受尊敬的专家及社会活动家的职 务提倡经证实的烟草控制方案。提倡医院和医疗机构无 烟应为医生的首要任务。作为人们试图改善自身健康状 况和接受疾病治疗的医疗中心, 允许吸烟的行为是完全 不合理的。

在中国解决吸烟的责任亦在于医生工作的机构和代 表医生的专业团体机构。医院和医疗机构的管理者和经 营者应该执行无烟环境、确保检查和记录、张贴政策， 以明示所有的吸烟患者并为医生提供他们戒烟所需的必 要支持, 比如提供持续的教育和培训。代表医生的专业 团体亦应是使其成员发生改变的载体, 告知医生最新关 于烟草相关危害的研究, 鼓励他们成为积极的公众模范 且不吸烟，而且利用他们作为专业机构的权威以提倡政 府的基于循证的烟草控制干预。

\section{哪些支持是可以采纳的?}

幸运的是，对医生、医疗卫生机构、决策制定者 和政府的大量指导有助于其执行基于循证的烟草控制方 案。WHO FCTC是拥有超过165个缔约方的多边条约, 旨 在对抗全球的烟草流行 ${ }^{[25]}$ 。FCTC为各国降低烟草供应和 烟草需求规划了一幅蓝图。中国批准履行了FCTC, 并因 此承诺通过共同抗击烟草来保护中国公民的健康。

在FCTC下, 许多指南已被采纳, 有助于各缔约方 履行其义务。他们提供了最佳的现有证据及已经成功执 行烟草控制方案的其它缔约方的经验。政府采纳的指南 是关于：防止烟草控制方面的公共卫生政策受烟草行业
的商业和其它既得利益的影响（公约第 5.3条）; 防止 接触烟草烟雾（公约第8条）; 烟草制品的包装和标签 (公约第 11 条) 和烟草广告、促销和赞助 ( 公约第 13 条) ${ }^{[26]}$ 。

关于教育、交流、培训和公众意识 ( 公约第12条) 和与烟草依赖和戒烟有关的降低烟草需求 (公约第 14 条）的指南也正在制定中。

2008年WHO全球烟草流行报告：MPOWER系列政 策包含了6项重要的烟草控制政策，这为执行FCTC提供 了进一步的指导 ${ }^{[27]}$ 。2008年报告首次全面地分析了全球 烟草使用状况和烟草控制的努力状况。2009年公布的第2 版MPOWER报告关注了无烟政策的执行情况 ${ }^{[28]}$ 。报告显 示，中国仍有待提供无烟的卫生保健机构。

2009年5月, 中国卫生部及其它相关部委印发了题 为“关于2011年起全国医疗卫生系统全面禁烟的决定”的 政策。决定提出了确保2011年实现卫生行政部门和医疗 卫生机构全面禁烟的策略和方案的具体工作计划 ${ }^{[29]}$ 。在 全国实现无烟示范医院的进程取得了缓慢进展, 这将为 其它国家的学习提供借鉴和培训场所 ${ }^{[30]}$ 。为了进一步协 助医生, 在2009年年底临床医生戒烟指南已被修订并重 新发布。该指南旨在协助医生明示吸烟者、诊断和治疗 烟草依赖以及提供及时的戒烟服务 ${ }^{[31]}$ 。在推进中国快速 实现卫生部规定的无烟政策及履行FCTC规定的义务中, 医生均发挥着巨大作用。

\section{总结}

治疗烟草相关疾病的医生, 尤其是治疗肺癌的医 生，在烟草控制中责无旁贷。首先应从他们自身的行为 和做法开始, 如果医生为吸烟者, 则应戒烟, 如果其继 续吸烟, 这提供了一个信息：医生可能并不相信吸烟有 害健康。积极开展关于肺癌患者戒烟的咨询是当地社区 传播信息的有效途径。努力实现医疗场所的无烟环境。 了解烟草控制机构开展的烟草宣传活动, 如果可能的话 予以支持。

医生对中国烟草控制的成功至关重要。他们积极参 与解决烟草使用过度的难题, 有助于预防许许多多可避 免的过早死亡。

\section{Acknowledgments}

Thank you to Marina Haritos, Lin Li and Indra Haslam

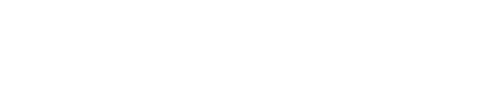


from the Cancer Council Victoria, Australia, for research assistance with this paper.

\section{参 考文 献}

1 World Health Organization, WHO Report on the Global Tobacco Epidemic, 2008: the MPOWER package [Internet]. Geneva: World Health Organization; c2008 [cited 2009 Dec 7]. Available from: http://www.who.int/tobacco/mpower/en/.

2 Liu BQ, Peto R, Chen ZM, et al. Emerging tobacco hazards in China: 1. Retrospective proportional mortality study of one million deaths. BMJ, 1998, 317(7170): 1411-1422.

3 Shafey O, Eriksen M, Ross $\mathrm{H}$, et al. The Tobacco Atlas: Third edition [Internet]. Atlanta, Georgia: American Cancer Society; c2009 [cited 2009 Dec 7]. Available from: http://www.cancer.org/docroot/AA/content/AA_2_5_9x _Tobacco_Atlas_3rd_Ed.asp?sitearea $=\&$ level=.

4 Yang G, Fan L, Tan J, et al. Smoking in China: findings of the 1996 National Prevalence Survey. JAMA, 1999, 282(13): 1247-1253.

5 Yang GH, Ma JM, Liu N, et al. Smoking and passive smoking in Chinese, 2002. Zhonghua Liu Xing Bing Xue Za Zhi, 2005, 26(2): 77-83. In Chinese.

6 World Health Organization, WHO Report on the Global Tobacco Epidemic, 2009: implementing smoke-free environments [Internet]. Geneva: World Health Organization [cited 2009 Dec 11]; [34. Implementation of effective measures is gaining momentum]. Available from: http://www.who.int/tobacco/mpower/2009/d_gtcr_implementing_effective_measures.pdf.

7 Doll R, Hill AB. Lung cancer and other causes of death in relation to smoking; a second report on the mortality of British doctors. BMJ, 1956, 2(5001): 1071-1076.

8 United States. Surgeon General's Advisory Committee on Smoking and Health. Smoking and Health: Report of the Advisory Committee to the Surgeon General of the Public Health Service [Washington]: United States. Public Health Service. Office of the Surgeon General; 1964.

9 Winstanley M. Tobacco in Australia: Facts and Issues [Internet]. Melbourne: Cancer Council Victoria; 2008 Chapter 3, The health effects of active smoking; [cited 2009 Dec 7]. Available from: http://www.tobaccoinaustralia.org. au/chapter-3-health-effects.

$10 \mathrm{He}$ J, Gu D, Wu X, et al. Major causes of death among men and women in China. N Engl J Med, 2005, 353(11): 1124-1134.

11 Gu D, Kelly TN, Wu X, et al. Mortality attributable to smoking in China. N Engl J Med, 2009, 360(2): 150-159.

12 Winstanley M. Tobacco in Australia: Facts and Issues [Internet]. Melbourne: Cancer Council Victoria; 2008 Chapter 4, The health effects of secondhand smoke; [cited 2009 Dec 7]. Available from: http://www.tobaccoinaustralia. org.au/chapter-4-secondhand.

13 U.S. Department of Health and Human Services. The health consequences of involuntary exposure to tobacco smoke: a report of the Surgeon General. Atlanta, Ga.: U.S. Department of Health and Human Services, Centers for Disease Control and Prevention, National Center for Chronic Disease Pre- vention and Health Promotion, Office on Smoking and Health; 2006. 709 p.

14 Gan Q, Smith KR, Hammond SK, et al. Disease burden of adult lung cancer and ischaemic heart disease from passive tobacco smoking in China. Disease burden of adult lung cancer and ischaemic heart disease from passive tobacco smoking in China. 2007; 16: 417-422.

15 Peto R, Chen ZM, Boreham J. Tobacco: The growing epidemic in China. CVD Prevention and Control, 2009, 4(1): 61-70.

16 Sung HY, Wang L, Jin S, et al. Economic burden of smoking in China, 2000. Tob Control, 2006,15(Suppl 1): i5-i11.

17 Jiang Y, Ong MK, Tong EK, et al. Chinese physicians and their smoking knowledge, attitudes, and practices. Am J Prev Med, 2007, 33(1): 15-22.

18 Li HZ, Sun W, Cheng F, et al. Cigarette smoking status and smoking cessation counseling of Chinese physicians in Wuhan, Hubei province. Asia Pac J Public Health, 2008, 20(3): 183-192.

19 Smith DR, Wei N, Zhang YJ, et al. Tobacco smoking habits among a crosssection of rural physicians in China. Aust J Rural Health, 2006, 14(2): 66-71.

20 Li HZ, Fish D, Zhou X. Increase in cigarette smoking and decline of antismoking counselling among Chinese physicians: 1987-1996. Health Promot Int, 1999, 14(2): 123-131.

21 Jiang Y, Li X, Wu X, et al. Smoking behaviour of Chinese physicians. Chin J Prev Contr Chron Dis, 2009, 17(3): 224-227.

22 Davis RM. When doctors smoke. Tob Control, 1993, 2: 187-188.

23 Smith DR, Leggat PA. The historical decline of tobacco smoking among Australian physicians: 1964-1997. Tob Induc Dis, 2008, 4: 13.

24 Stead LF, Bergson G, Lancaster T. Physician advice for smoking cessation. Cochrane Database of Syst Rev 2008, Issue 2. Art No.:CD000165. DOI. 10.1002/14651858.CD000165.pub3.

25 Further information available from: http://www.who.int/fctc/ text_download/en/index.html

26 Further information available from: http://www.who.int/fctc/guidelines/ en/

27 Further information available from: http://www.who.int/tobacco/mpower/2008/en/index.html

28 Further information available from: http://www.who.int/tobacco/mpower/2009/en/

29 "Ministry of Health's notice on comprehensive smoking ban in the medical and health system from 2011". Chin J Prev Contr Chron Dis, 2009, 17(3): 223.

30 Troedsson H, speaker. Speech at Double Ten Initiative Launch Ceremony [Internet]. Beijing, China: WHO Representative Office in China; 2009 Dec 1 [cited 2009 Dec 11]. Available from: http://www.wpro.who.int/china/ media_centre/speeches/speech_20090301.htm.

31 "The new version of China Clinical Smoking Cessation has been completed", from the 7/12/2009 The Health Daily. Accessed on 8 January 2010 at the following website: http://www.jkb.com.cn/document/106180. $\mathrm{htm}$ ? docid $=106180 \& \mathrm{cat}=0 \mathrm{G} 1 \& \mathrm{sKeyWord}=$ null.

(本文编辑 周玲) 


\section{University Library}

\section{- M M I E R R V A gateway to Melbourne's research publications}

Minerva Access is the Institutional Repository of The University of Melbourne

Author/s:

Lindorff, KJ;Hill, DJ

Title:

[Physicians' role in advancing tobacco control in China].

Date:

2010-06

Citation:

Lindorff, K. J. \& Hill, D. J. (2010). [Physicians' role in advancing tobacco control in China].. Zhongguo Fei Ai Za Zhi, 13 (6), pp.632-636. https://doi.org/10.3779/ j.issn.1009-3419.2010.06.012.

Persistent Link:

http://hdl.handle.net/11343/250402

License:

CC BY 